\title{
Homologous recombination-mediated targeted integration in monkey embryos using TALE nucleases
}

Chu Chu ${ }^{1 \dagger}$, Zhaohui Yang ${ }^{1 \dagger}$, Jiayin Yang ${ }^{2}$, Li Yan $^{1}$, Chenyang Si ${ }^{1}$, Yu Kang ${ }^{1}$, Zhenzhen Chen ${ }^{1}$, Yongchang Chen ${ }^{1}$, Weizhi $\mathrm{Ji}^{1}$ and Yuyu Niu${ }^{1 *}$

\begin{abstract}
Background: Non-human primate (NHP) models can closely mimic human physiological functions and are therefore highly valuable in biomedical research. Genome editing is now developing rapidly due to the precision and efficiency offered by engineered site-specific endonuclease-based systems, such as transcription activator-like effector nucleases (TALENS) and the clustered regularly interspaced short palindromic repeats (CRISPR)/CRISPRassociated protein-9 nuclease (Cas9) system. It has been demonstrated that these programmable nucleases can introduce genetic changes in embryos from many species including NHPs. In 2014, we reported the first genetic editing of macaques using TALENs and CRISPR/Cas9. Subsequently, we characterized the phenotype of a methyl CpG binding protein 2 (MECP2)-mutant cynomolgus monkey model of Rett syndrome generated using the TALEN approach. These efforts not only accelerated the advance of modeling genetic diseases in NHPs, but also encouraged us to develop specific gene knock-in monkeys. In this study, we assess the possibility of homologous recombination (HR)-mediated gene replacement using TALENs in monkeys, and generate preimplantation embryos carrying an EmGFP fluorescent reporter constructed in the OCT4 gene.

Result: We assembled a pair of TALENs specific to the first exon of the OCT4 gene and constructed a donor vector consisting of the homology arms cloned from the monkey genome DNA, flanking an EmGFP cassette. Next, we coinjected the TALENs-coding plasmid and donor plasmid into the cytoplasm of 122 zygotes 6-8 $\mathrm{h}$ after fertilization. Sequencing and immunofluorescence revealed that the OCT4-EMGFP knock-in allele had been successfully generated by TALENs-mediated HR at an efficiency of $11.3 \%$ (7 out of 62 ) or $11.1 \%$ (1 out of 9), respectively, in monkey embryos.
\end{abstract}

Conclusion: We have successfully, for the first time, obtained OCT4-EmGFP knock-in monkey embryos via HR mediated by TALENs. Our results suggest that gene targeting through TALEN-assisted HR is a useful approach to introduce precise genetic modification in NHPs.

Keywords: Non-human primate, Homologous recombination, TALENs, Gene editing

\section{Background}

Although a wide range of organisms, including worms, fruit flies, zebrafish, and rodents, have been used as research tools for most discoveries [1], non-human primates (NHPs) are excellent models to study human disease and develop therapeutic strategies because of

\footnotetext{
* Correspondence: niuyy@lpbr.cn

${ }^{+}$Chu Chu and Zhaohui Yang contributed equally to this work.

YYunnan Key Laboratory of Primate Biomedicine Research; Institute of

Primate Translational Medicine, Kunming University of Science and

Technology, Kunming 650500, China

Full list of author information is available at the end of the article
}

their genetic, physiological and neurological similarity to humans [1-5]. Over the years, genetic approaches have been widely applied to model organisms. However, genetic engineering in monkeys was not available until 2001, when retrovirus-based methods were successfully used to generate transgenic monkey models [6]. This seminal work demonstrated that the monkey genome could be genetically modified efficiently. Subsequently, taking advantage of the high efficiency of lentiviral infection, the groups of $\mathrm{Li}$ and Sasaki successfully obtained several transgenic monkeys carrying the mutant $H T T$

(c) The Author(s). 2019 Open Access This article is distributed under the terms of the Creative Commons Attribution 4.0 International License (http://creativecommons.org/licenses/by/4.0/), which permits unrestricted use, distribution, and 
and GFP transgenes, respectively [7, 8]. However, while lentiviral transduction has been a powerful and efficient tool in genetic modification of animal genomes, it has often failed to achieve precise editing. The 2011 emergence of new-generation genome-editing methods based on reprogrammable, site-specific endonucleases, especially the clustered regularly interspaced short palindromic repeats (CRISPR)/CRISPR-associated protein-9 nuclease (Cas9) system and transcription activator-like effector nuclease (TALEN), made genome engineering more effective and reliable. Relative construction simplicity as well as precise and efficient genetic modification of target sites associated with these technologies has dramatically advanced genome editing in diverse species $[9,10]$. In particular, these methods substantially facilitated the generation of non-human primate (NHP) models of human diseases. Until now, a number of such models have been established, including those of autism spectrum disorder (ASD) [11], Huntington's disease
(HD) [7], Parkinson's disease (PD) [12], Duchenne muscular dystrophy (DMD) [13], and, most recently, Rett syndrome [3].

TALENs are fusions of the endonuclease domain consisting of the FokI restriction enzyme, which is only active as a dimer, and the engineered DNA-binding domain comprised by TAL effector (TALE) repeats. These highly conserved repeats are derived from naturally-occurring TALEs of the plant pathogen Xanthomonas, which contribute to disease or trigger defense by binding the host DNA and activating effector-specific host genes [14] .

TALENs can easily modify genomic DNA by introducing double-strand breaks (DSBs) at the desired site, stimulating the cellular DNA repair system. The main components of this system are non-homologous end-joining (NHEJ) and homologous recombination (HR) that result in knock-outs and knock-ins, respectively. NHEJ is a stochastic and error-prone repair process that does not require a repair template and

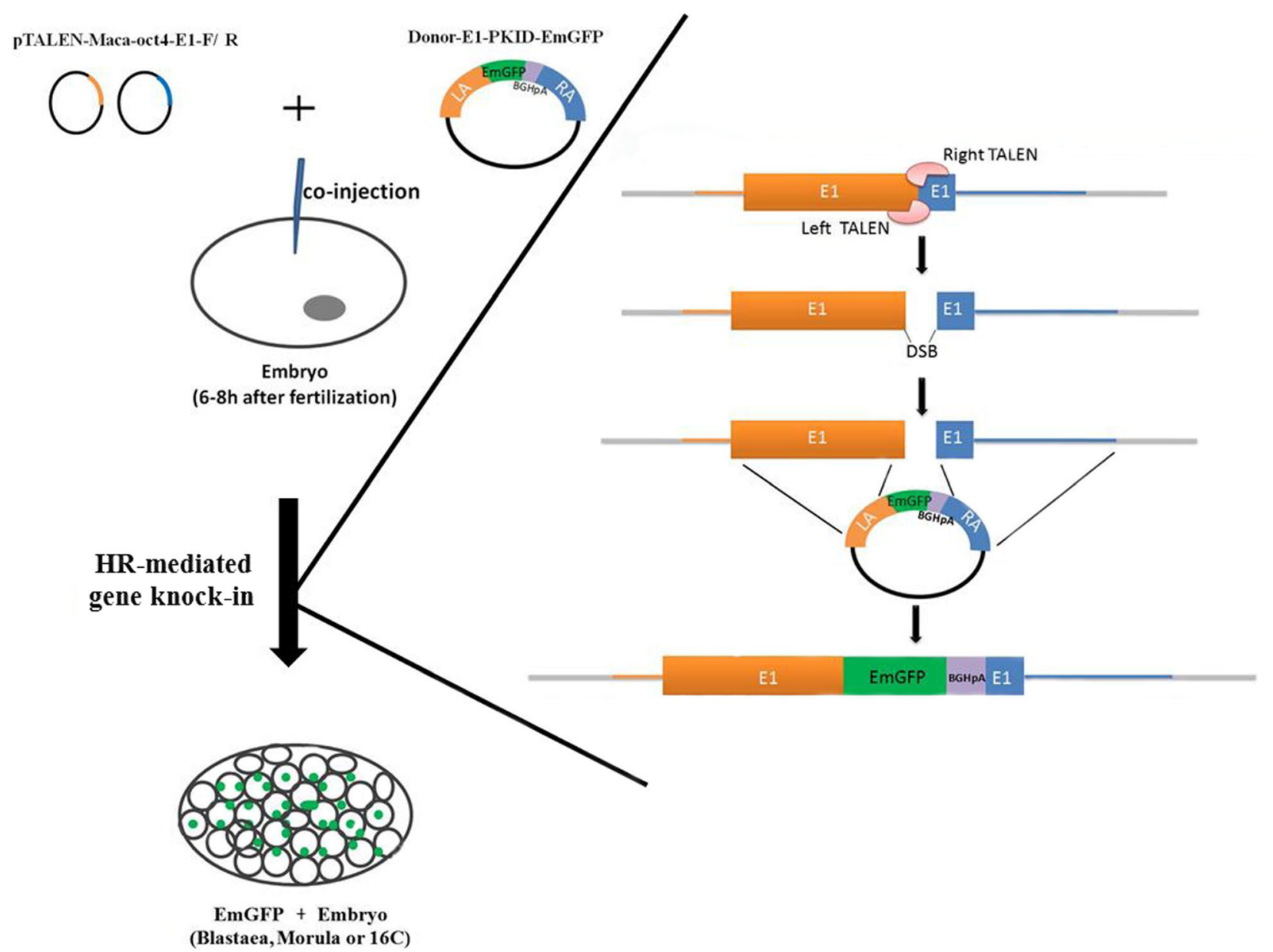

Fig. 1 Workflow of TALEN-mediated generation of a monkey embryo carrying an EmGFP reporter in the OCT4 gene. TALENs-Coding plasmids, PTALEN-Maca-oct4-E1-F/R, and the donor vector Donor-E1-PKID-EmGFP that targets exon 1 of the OCT4 gene were designed and co-injected into the cytoplasm of a zygote $6-8 \mathrm{~h}$ after fertilization. Treated embryos at the blastocyst, morula and 16-cell stages were collected and analyzed 


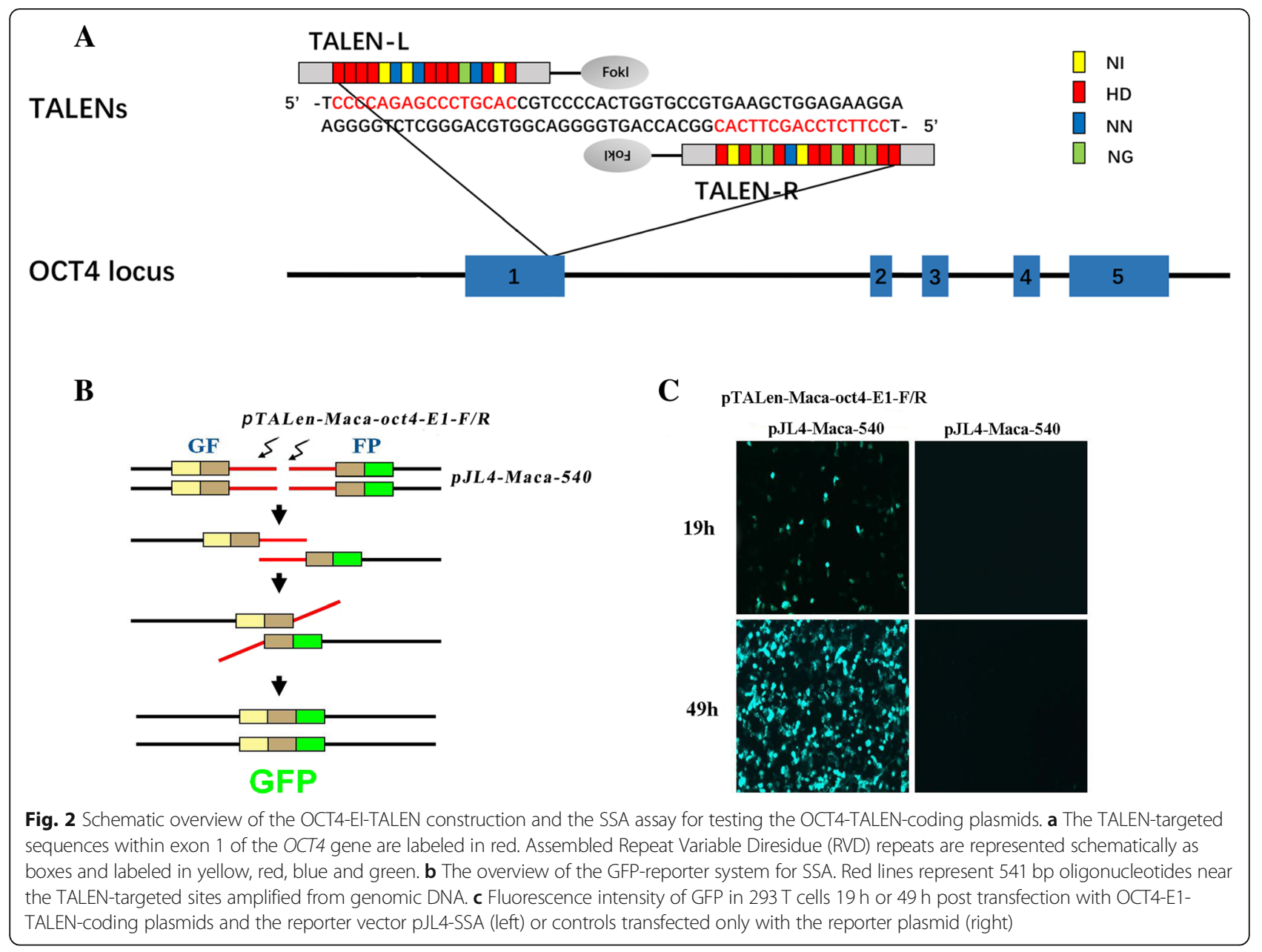

introduces random small insertions or deletions at DNA breakpoints [15]. Gene editing by this system has been used in mammalian cells to disrupt genes, delete chromosomal segments, or restore aberrant reading frames [14]. $\mathrm{HR}$, on the other hand, is a precise repair pathway that requires a DNA template, which harbors a homologous sequence corresponding to the genomic DNA at the DSB site. HR has been used to correct certain mutations associated with diseases such as epidermolysis bullosa [16], sickle cell disease [17, 18], beta-thalassemia [19], and alpha-1 antitrypsin deficiency [20]. The efficiency of gene correction strategies based solely on HR is closely linked to the genomic target, cell type, cell-cycle state, organism species, and efficient delivery of an exogenous repair template. Unlike NHEJ, which occurs during the whole cell cycle, HR is only active during the late S/G2 phases, making HR-dependent gene knock-ins more difficult to achieve compared to the gene knock-outs mediated by NHEJ. Currently, HR-mediated knock-ins have been generated in many species including zebrafish [21], mice [22], rabbit [23], goat [24], sheep [25], cattle [26] and pig [27]. Recently, the groups of Yang and Niu successfully used CRISPR/Cas9-assisted HR to obtain knock-in cynomolgus monkeys with a reporter gene system $[28,29]$. Here, we demonstrate TALEN-assisted, HR-mediated generation of monkey preimplantation embryos carrying an EmGFP fluorescent reporter constructed in the OCT4 gene (Fig. 1). Our results demonstrate that TALENbased HR is a feasible approach to generate knock-in NHP models.

Table 1 In vitro developmental potential of the TALENs-injected embryos

\begin{tabular}{|c|c|c|c|c|c|c|}
\hline \multirow[t]{2}{*}{ Group } & \multirow{2}{*}{$\begin{array}{l}\text { Number } \\
\text { of } \\
\text { embryos }\end{array}$} & \multicolumn{5}{|c|}{ Development stage reached (\%) } \\
\hline & & 2-cell & 8-cell & 16-cell & morula & blastocyst \\
\hline Injected & 122 & $115(94.3)$ & $96(78.7)$ & $71(58.2)$ & $57(47.7)$ & $49(40.2)$ \\
\hline Control & 34 & $34(100)$ & $30(88.2)$ & $23(67.64)$ & $17(50)$ & $13(38)$ \\
\hline
\end{tabular}




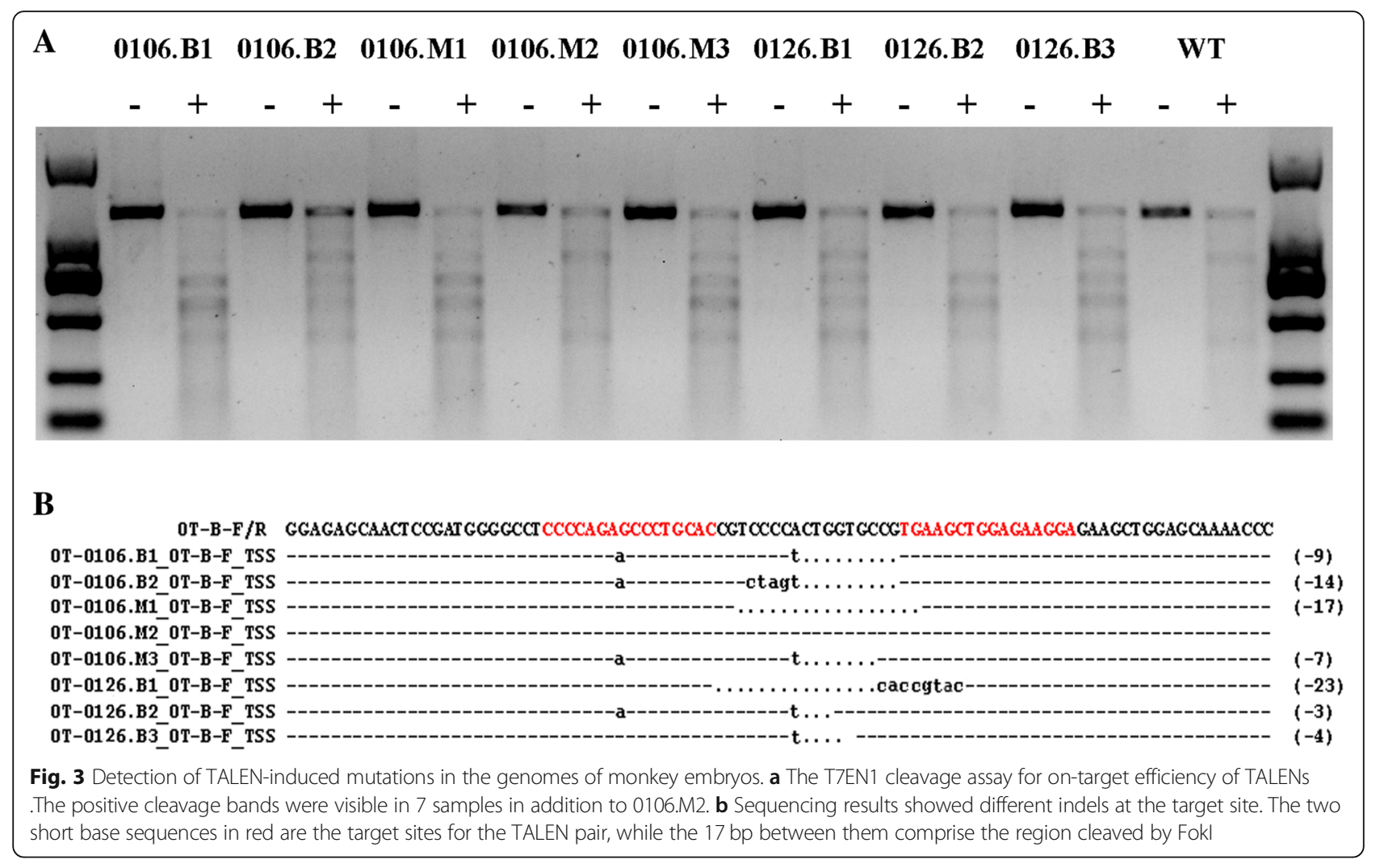

\section{Results}

Construction of TALENs and evaluation of their activity in human cells

The monkey OCT4 gene contains 5 exons, similar to that of humans and mice (monkey OCT4 Gene ID: 714760, human OCT4 Gene ID: 5460, mice OCT4 gene ID: 18999, from https://www.ncbi.nlm.nih.gov/
gene/?term=OCT4). We designed a pair of TALEN constructs to target exon 1 (Fig. 2a), which is the efficient site for inserting a reporter gene to indicate the expression of OCT4 [30, 31]. These sites are conserved in humans, so we initially used the single strand annealing (SSA) assay in $293 \mathrm{~T}$ cells to evaluate the cutting efficiency of Oct4-E1-TALENs based on the GFP expression level,

Table 2 Primers used for genotyping

\begin{tabular}{|c|c|c|c|}
\hline Primer & Sequence (5' to $3^{\prime}$ ) & Usage & Amplicon(bp) \\
\hline L1-F & AGGATCGCTTGAGGACAGGAATT & PCR & $3259 \mathrm{bp}\left(5^{\prime} \mathrm{gDNA} \sim 3^{\prime} \mathrm{gDNA}\right)$ \\
\hline L1-R & GGAGCCATCACAGGAGACAGAAAA & PCR/sequencing & \\
\hline S1-F & GATGCATTGAGGGATAGCTCCACACACACATTC & PCR/sequencing & 1795 bp (5' gDNA EmGFP) \\
\hline $\mathrm{S} 1-\mathrm{R}$ & AGGACCATGTGATCGCGCTTCT & PCR & \\
\hline S2-F & CAGAAGAACGGCATCAAG & PCR & 2109 bp (EmGFP 3'gDNA) \\
\hline $\mathrm{S} 2-\mathrm{R}$ & AGTAGGGAGGAAGCAGTAGAA & PCR & \\
\hline$G-F$ & CACAAGTTCAGCGTGTCCG & $P C R$ & $421 \mathrm{bp}($ EmGFP) \\
\hline$G-R$ & AGTTCACCTTGATGCCGTTC & PCR & \\
\hline LI-W1R-G08 & ATCCCTCAGCAGCTCTACATTT & sequencing & \\
\hline L1-W2R-H11 & CAGCTTCACGGCACCAGTAC & sequencing & \\
\hline L1-W3R-C04 & GTAGCGGGCGAAGCACTG & sequencing & \\
\hline S12-W1R-C01 & TCCGAGGATCAACCCAGC & sequencing & \\
\hline OT-F & CACCACCATTAGGCAAAC & PCR & 1201 bp target site included \\
\hline OT-R & GAGGCTGAAGTCAATCAAAA & PCR & \\
\hline
\end{tabular}




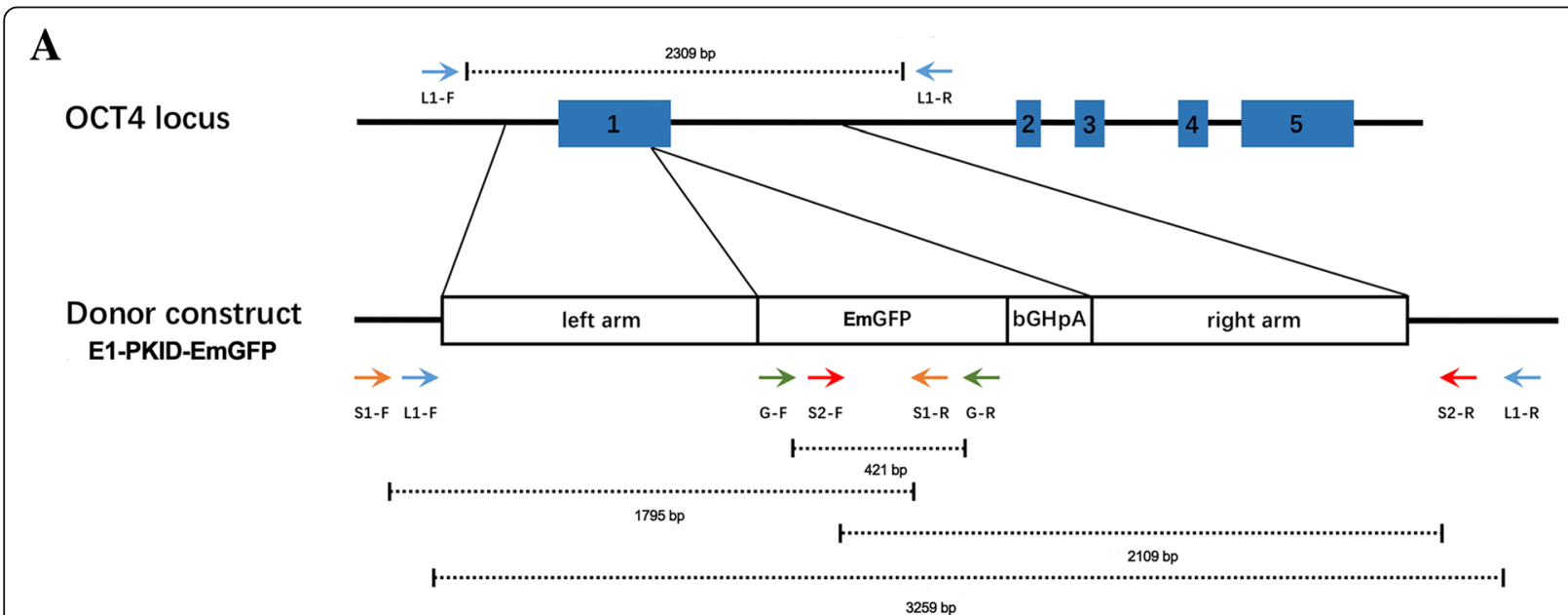

B

5' gDNA
LA EmGFP bGHpA RA 3' gDNA
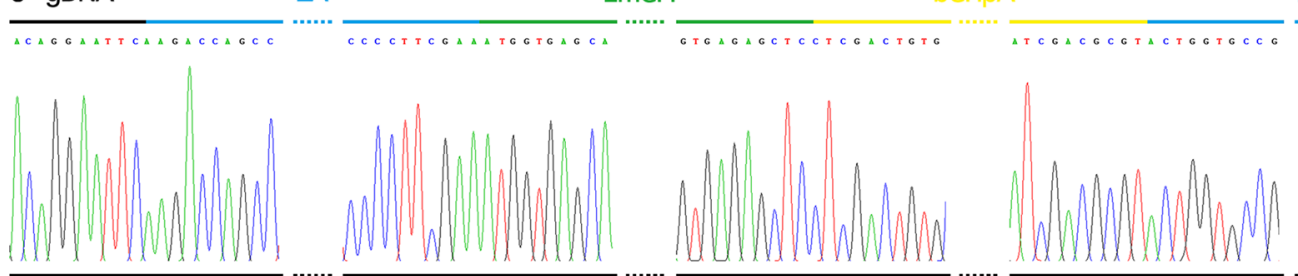

CTTTATACATCATTACCTCA

C

D
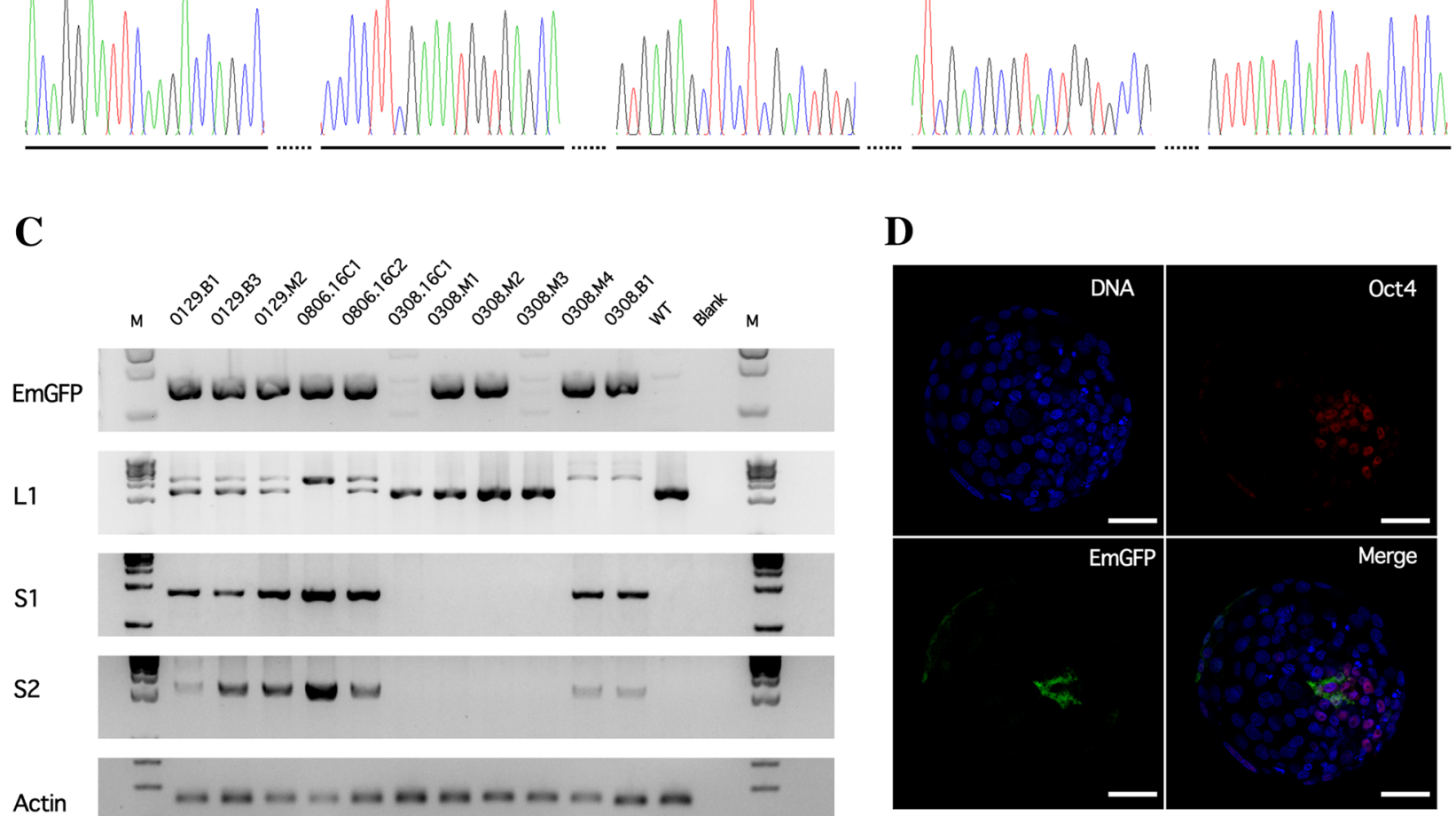

L1

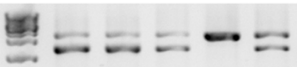

S1

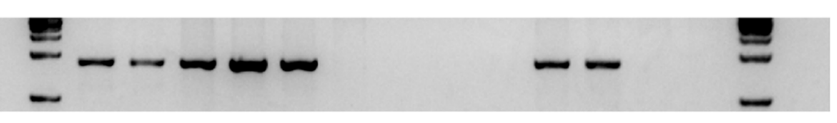

S2

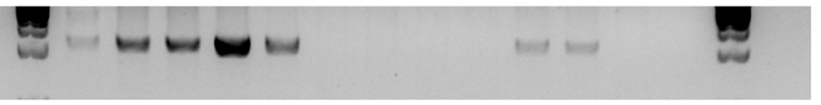

Actin

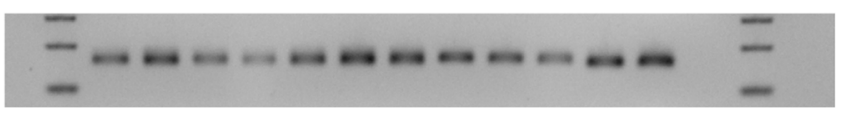

Targeted Blastocyst

Fig. 4 Genotyping and immunofluorescence of embryos generated by TALEN-mediated genome engineering. a Schematic overview of the strategy to generate an OCT4-EmGFP knock-in allele. The homologous arms of the donor vector are indicated as LA (839 bp) and RA (1001 bp). PCR primers used for PCR genotyping are shown as arrows in different colors. $\mathbf{b}$ PCR products obtained using primers L1-F and L1-R were sequenced. Sequence across the targeted region confirmed the correct fusion of EmGFP to the first exon of OCT4. c EmGFP: PCR genotyping using primers G-F and G-R produced bands of 421-bp fragment in the samples from nine embryos, suggesting the possible insertion or precise knock-in of the EmGFP gene. L1: PCR genotyping using primers L1-F and L1-R produced large products (3259 bp) in seven embryo samples, indicating the EmGFP sequence was integrated. The samples 0806.16C1, $0308 \mathrm{M} 4$ and 0308.B1 only contain the larger product, suggesting either both alleles were targeted, or one allele failed to amplify. S1 and S2: PCR genotyping using primers S1-F and S1-R, S2-F and S2-R produced bands with correct size (1795 bp and $2109 \mathrm{bp}$ ) in the samples from the targeted embryos. $\mathbf{d}$ Immunostaining of targeted blastocysts using anti-GFP antibody showed a signal in the ICM. Scale bar, $50 \mu \mathrm{M}$ 
Table 3 The positive rates of EmGFP knock-in demonstrated by PCR analysis or GFP detection

\begin{tabular}{llll}
\hline Method & $\begin{array}{l}\text { Number of zygote } \\
\text { injected with plasmid }\end{array}$ & $\begin{array}{l}\text { Number of } \\
\text { positive embryos }\end{array}$ & positive rate \\
\hline PCR analysis & 62 & 7 & $11.3 \%$ \\
GFP detection & 9 & 1 & $11.1 \%$ \\
\hline
\end{tabular}

namely the percentage of GFP positive cells (Fig. 2b). Expression of GFP could be observed in $293 \mathrm{~T}$ cells transfected with the Oct4-E1-TALEN-coding plasmids, pTALEN-Maca-oct4-E1-F /R, and the reporter vector pJL4-Maca-540 both after $19 \mathrm{~h}$ and $49 \mathrm{~h}$, but not in the control group transfected only with the reporter plasmid (Fig. 2c). These results showed that the TALENs were designed and constructed to effectively induce DSBs at the target sites in the human genome, suggesting that this pair of TALENs would work in a monkey as well because of high genomic homology.

\section{Development of monkey embryos microinjected with the constructed plasmids}

The plasmids coding for TALENs, pTALEN-Maca-oct4E1-F /R, and the donor vector, Donor-E1-PKID-EmGFP, were mixed and injected into the cytoplasm of 122 zygotes 6-8 $\mathrm{h}$ after fertilization using a microinjection system under standard conditions. Based on the number of zygotes that were successfully injected, 94.3\% (115 of 122), 78.7\% (96 of 122), $58.2 \%$ (71 of 122), $47.7 \%$ (57 of 122), and $40.2 \%$ (49 of 122) of the TALENs-treated embryos progressed to the 2-cell, 8-cell, 16-cell, morula, and blastocyst stage, respectively (Table 1). The developmental competence of embryos injected with TALENs was normal, judging by the percentage of embryos that reached the blastocyst stage (40.2\%). These results are similar to our previous report for those embryos derived from in vitro fertilization (IVF) [2], indicating that this system is safe and does not influence the development of NHP embryos.

\section{Genotyping to confirm the targeted gene integration}

To test the efficiency of TALENs, the genomic DNA was isolated from the embryos with normal development at the 16-cell, morula and blastocyst stages, respectively, and screened for the presence of site-specific gene modification by PCR amplification of the regions surrounding the target sites, as well as by the T7EN1 cleavage assay (Fig. 3a). The PCR products corresponding to the target sites were amplified using the OT-F/R primers (Table 2) from individual embryos $(n=8)$ in the morula or blastocyst stage and then sequenced. The genomes of up to $87 \%$ (7/8) of monkey embryos injected with TALEN-coding plasmids were modified at the target site, visualized by the cleavage bands in the target gene (Fig. 3a). Further characterization of the cleavage by sequencing indicated different indels at the target site with variable mutation size (Fig. 3b). This data demonstrated that the TALENs could also function effectively in monkey embryos.

To determine whether the EmGFP sequence was inserted into the target OCT4 locus, four primers (Fig. 4a, Table 2) were used to amplify the different PCR products from the individual embryos $(n=62)$ in the 16-cell, morula or blastocyst stages, and these genes were then sequenced. PCR using a pair of primers for $\operatorname{EmGFP}(\mathrm{G}-\mathrm{F} / \mathrm{R})$ amplified a specific band in the genomic DNA of the embryos (Fig. 4c), which suggested the possible insertion or precise knock-in of the EmGFP gene. Using the forward primer outside of the left homology arm (LA) region (L1-F) and the reverse primer outside the right homology arm (RA) region (L1-R), we detected a large PCR product $(3259 \mathrm{~kb})$ for the EmGFP-knock-in in the OCT4 gene locus of seven embryos (Fig. 4c). The PCR products of samples $0806.16 \mathrm{C} 1$ and $0806.16 \mathrm{C} 2$ were sequenced, and both $5^{\prime}$ and $3^{\prime}$ boundaries between OCT4 and EmGFP were detected as designed (Fig. 4b, Additional file 1). To further confirm the precise modification, we were then able to amplify a fragment of the expected size from the seven EmGFP-positive embryos using a forward primer outside of the LA region (S1-F) with an EmGFP-specific reverse primer (S1-R), and an EmGFP-specific forward primer (S2-F) with a reverse primer outside of the RA region (S2-R) (Fig. 4c). These results of electrophoresis and sequencing demonstrated that the OCT4-EmGFP knock-in allele has been successfully generated by TALENs-mediated HR at the efficiency of $11.3 \%$ (7 out of 62 ) in monkey embryos (Table 3 ).

\section{Detection of OCT4 and GFP by immunofluorescence}

In order to investigate the function of the knock-in EmGFP as a reporter for OCT4 expression, OCT4 and GFP were assayed by immunofluorescence in the individual blastocysts $(n=9)$. As expected based on a correctly targeted and functional allele, EmGFP expression was observed in the partial inner cell mass (ICM) region of the targeted blastocysts (Fig. 4d) at an efficiency of $11.1 \%$ (1 out of 9), corresponding with the value obtained by sequencing (Table 3). Besides, the proportion of the EmGFP-positive blastomere in OCT4-positive blastomere is $50 \%(11 / 20)$. These results indicated that, although with mosaicism, EmGFP was inserted into the desired site and could also successfully report the expression of OCT4.

Assessment of the specificity of TALEN-mediated cleavage To test the specificity of the cleavage by TALENs, seven highly-ranked potential off-target sites for OCT4 TALENs were predicted and selected (Additional file 2: Table S1). The off-target effects were comprehensively assessed in the same way as the on-target effect, using genomic DNA from 10 embryos that were modified at the target site. 
Thus, the fragments around the seven potential off-target gene loci were amplified by PCR and sequenced. Sequencing results (Additional file 3) showed that no off-target mutations were found at the predicted sites of genomic DNA from the TALEN-injected embryos, suggesting that the TALENs we designed and constructed had high specificity and thus were sufficiently safe to use in monkeys.

\section{Discussion}

With the emergence of reliable and precise gene editing tools in the recent years, gene modification has been dramatically brought to a new level. Among the engineered site-specific nucleases, CRISPR/Cas9 system has developed most rapidly and is now the most widely-used tool in research labs because of its construction simplicity and high efficiency. However, this system is still controversial in terms of safety because of the associated frequency of off-target effects [32, 33]. In comparison, TALEN is safer to use due to its requirement for being a dimer to be active and because it consists only of proteins. Therefore, TALEN is still an important and attractive genetic tool for functional genomics. In this study, we used the classic method of Golden Gate cloning for TAL assembly to construct a TALEN pair that could effectively work in both humans and monkeys at exon 1 of the OCT4 gene (Fig. 2, Fig. 3).

Mammalian animal models have contributed significantly to studies in both basic biology and human diseases. In this regard, genome engineering in mammals is highly relevant, especially targeted gene knock-in methods. For example, allowing reporter genes to be successfully inserted into specific genes important in embryonic stem cell mechanisms, such as OCT4, NANOG and SOX2, can facilitate developmental biology research [27] [34]. Furthermore, insertion of certain functional genes can be helpful in the generation of animal models or in animal husbandry [3, 24]. In addition, gene knock-in could be used to replace the endogenous base sequences for repairing mutations. Many knock-in animals of different species have been generated to date. In this work, we obtained monkey embryos with precise EmGFP knock-in at the OCT4 gene locus by TALEN-mediated HR at an efficiency of $11 \%$ (Fig. 4, Table 3), which corresponds to the general achievable efficiency of gene knock-in in mammalian systems [22] [35]. According to the results of immunofluorescence experiments, detection of GFP not only confirmed the expression of OCT4, but also reliably indicated the ICM regions of blastocysts. Therefore, this system could be a useful tool for embryonic development research in monkeys.

NHPs are the most suitable models to study human disease because of their high homology to humans. Our results are significant for the genetic modification research in monkeys, and are therefore highly valuable for the construction of reliable human disease models. Similar to the work of Yang and Niu groups on gene knock-in monkeys using CRISPR/Cas9 system [28, 29], our results confirm the successful gene insertion but show a degree of mosaicism, indicated by the immunofluorescence result (Fig. 4d) and electrophoretic images from four samples, 0129.B1, 0129.B3, 0129.M2 and 0806.16C2. Nevertheless, there are also three genetically modified samples (0806.16C1, $0308 \mathrm{M} 3$ and 0308B1) that seem to be homozygous (Fig. 4c), encouraging us to undertake further study to overcome mosaicism in monkey genome editing.

Recently, J.R. et al. [36] combined 2C-HR-CRISPR with a modified biotin-streptavidin system to localize the repair templates to target sites, resulting in a morethan-tenfold increase (up to 95\%) in knock-in efficiency over standard methods. Although their result was based on the CRISPR/Cas9 system unlike our study, it prompts us to carefully consider the time window for the injection of the TALEN pair and the donor template. The structure of chromatin is one of the determining factors for achieving high-efficiency of gene knock-in. In this research, we injected TALENs-coding plasmids and the donor vector 6$8 \mathrm{~h}$ after fertilization, when chromatin is most opened. Therefore, in the future, we need to find the optimum conditions of introducing the editing system to obtain more efficient gene knock-in results in monkeys.

\section{Conclusions}

In this work, for the first time, we have successfully obtained OCT4-EmGFP knock-in monkey embryos by TALEN-assisted HR. Even though mosaicism was detected, the GFP could faithfully report the expression of OCT4. Our results suggest that gene targeting through HR mediated by TALENs is a feasible approach to introduce precise genetic modification in NHPs.

\section{Methods \\ Construction of TALEN-coding plasmids and the donor plasmid}

TALEs were designed using online software (https:// tale-nt.cac.cornell.edu/node/add/talen). Repeat Variable Diresidue (RVD)-containing repeats were assembled using the Golden Gate TAL Assembly Kit, and then were sub-cloned into FokI nuclease expression vectors containing modified ELD/KKR domains, ptCMV-153/ 47-VR-HD (Addgene \#50703). Assembled RVD repeats were as follows: HD HD HD HD NI NN NI NN HD HD HD NG NN HD NI HD for the left TALEN targeting CCCCAGAGCCCTGCAC; HD HD NG NG HD NG HD HD NI NN HD NG NG HD NI HD for the right TALEN targeting CCTTCTCCAGCTTCAC.

HR donor vector E1-PKID-EmGFP was constructed by standard molecular-cloning methods. For the OCT4 locus, the donor vector consists of the 839-bp 
5'-homology arm and the 1001-bp 3'-homology arm cloned from monkey (050139) gDNA, flanking an EmGFP cassette followed by the BGH polyA signal (Fig. 4, Additional file 4: Figure S1).

\section{SSA assay in human cells}

For construction of the reporter plasmid pJL4-Maca-540, the 541-bp oligonucleotides near the TALENs target site was amplified with primer Maca-540-F1/R1 (Additional file 5) and cloned into the backbone vector pJL4-SSA(Cell Inspire Bio)linearized with BglII and EcoRI (NEB, R0144 and R0101). In this way the GFP-coding sequence could be separated into two parts, GF and FP. The two parts could be annealed to express intact GFP only following successful application of TALENs. The TALEN plasmids and the reporter plasmids were transfected into HEK293T cells using polyethylenimine (PEI,Polysciences 23,966-2) by applying standard protocol [37]. At $19 \mathrm{~h}$ and $47 \mathrm{~h}$ post transfection, respectively, the fluorescence intensity of GFP was measured using confocal microscopy.

\begin{abstract}
Animals
Healthy female cynomolgus monkeys (Macaca fascicularis), ranging in age from 5 to 8, were selected for use in this study. All animals were housed at Yunnan Key Laboratory of Primate Biomedical Research (LPBR). These animals were only used for oocytes collection. After a seven-day observation period, no euthanasia needed, these animals returned to the outdoor animal colony of LPBR.
\end{abstract}

\section{Embryo collection}

Embryo collection and transfer were performed as previously described [38]. In brief, 11 healthy female cynomolgus monkeys aged 5-8 years with regular menstrual cycles were selected as oocyte donors for superovulation, which was performed by intramuscular injection with rhFSH (recombinant human follitropin alfa, GONAL-F, Merck Serono) for 8 days, then rhCG (recombinant human chorionic gonadotropin alfa, OVIDREL, Merck Serono) on day 9. 32$35 \mathrm{~h}$ after rhCG administration, Atropine $(0.02 \mathrm{mg} / \mathrm{kg}$,Tianjin Pharmaceutical Group, China), Benzyl penicillin sodium (20 mg/kg, Harbin Pharmaceutical Group, China) and morphine hydrochloride $(0.2 \mathrm{mg} / \mathrm{kg}$, Northeast Pharmaceutical Group, China) were intramuscular administered for preparing for minimally invasive surgery. Animals were anesthetized using Ketamine $(10 \mathrm{mg} / \mathrm{kg}$, Jiangsu Zhongmu Beikang Pharmaceutical CO., LTD, China).Oocytes were collected by laparoscopic follicular aspiration. Mature oocytes(MII oocytes,first polar body present) were used to perform intracytoplasmic sperm injection (ICSI) and the fertilization was confirmed by the presence of two pronuclei. The zygotes(including those subsequently microinjected with plasmids and) were cultured in the chemically defined, protein-free hamster embryo culture medium-10 (HECM-10) containing 10\% fetal calf serum (Hyclone Laboratories, SH30088.02) at $37^{\circ} \mathrm{C}$ in $5 \% \mathrm{CO} 2$.

\section{Microinjection of plasmids}

TALENs-coding plasmids (50 ng/ $\mu \mathrm{l})$ and donor plasmid $(10 \mathrm{ng} / \mu \mathrm{l})$ were mixed and microinjected into the cytoplasm of zygotes $6-8 \mathrm{~h}$ after fertilization using a microinjection system under standard conditions. The zygotes were then cultured in the chemically defined, protein-free hamster embryo culture medium-10 (HECM-10) containing $10 \%$ fetal calf serum (Hyclone Laboratories, $\mathrm{SH} 30088.02)$ at $37^{\circ} \mathrm{C}$ in $5 \% \mathrm{CO} 2$.

\section{Genomic DNA extraction and genotyping by PCR}

The individual embryos at the stage of 16-cell, morula or blastocyst were collected, and their genomic DNA was amplified using REPLI-g Single Cell Kit (QIAGEN, 150345). All PCR experiments were performed with Takara PrimeSTAR GXL DNA Polymerase (Takara, $\mathrm{R} 050 \mathrm{~B})$, and the products were analyzed by sequencing. Primers OT-F/R were used to amplify the target site region at the following conditions: $95^{\circ} \mathrm{C}$ for $5 \mathrm{~min}$; 35 cycles of $95^{\circ} \mathrm{C}$ for $30 \mathrm{~s}, 58^{\circ} \mathrm{C}$ for $30 \mathrm{~s}, 72^{\circ} \mathrm{C}$ for 1 min; $72{ }^{\circ} \mathrm{C}$ for $10 \mathrm{~min}$. Primers G-F/R localizing to EmGFP were used to confirm possible insertion of $E m G F P$, under the following conditions: $95^{\circ} \mathrm{C}$ for 5 min; 35 cycles of $95^{\circ} \mathrm{C}$ for $30 \mathrm{~s}, 56^{\circ} \mathrm{C}$ for $30 \mathrm{~s}, 72^{\circ} \mathrm{C}$ for $30 \mathrm{~s} ; 72^{\circ} \mathrm{C}$ for $5 \mathrm{~min}$. Primers $\mathrm{S} 1-\mathrm{F} / \mathrm{R}$ amplified the fragment over outside the LA region and EmGFP under the conditions: $95^{\circ} \mathrm{C}$ for $3 \mathrm{~min} ; 16$ cycles of $95^{\circ} \mathrm{C}$ for $30 \mathrm{~s}, 66^{\circ} \mathrm{C}$ for $30 \mathrm{~s}, 72^{\circ} \mathrm{C}$ for $1.5 \mathrm{~min},-0.5^{\circ} \mathrm{C} /$ cycle; 30 cycles of $95^{\circ} \mathrm{C}$ for $30 \mathrm{~s}, 58^{\circ} \mathrm{C}$ for $30 \mathrm{~s}, 72^{\circ} \mathrm{C}$ for $1.5 \mathrm{~min}$; $72^{\circ} \mathrm{C}$ for $5 \mathrm{~min}$. Primers S2-F/R amplified the fragment over outside the RA region and EmGFP under the conditions: $95^{\circ} \mathrm{C}$ for $5 \mathrm{~min} ; 35$ cycles of $95^{\circ} \mathrm{C}$ for $30 \mathrm{~s}, 54^{\circ} \mathrm{C}$ for $30 \mathrm{~s}, 72{ }^{\circ} \mathrm{C}$ for $2 \mathrm{~min} ; 72^{\circ} \mathrm{C}$ for $5 \mathrm{~min}$. Primers L-F/R detected the large PCR product for the intact EmGFP knock-in at the OCT4 locus, under the following conditions: $95^{\circ} \mathrm{C}$ for $5 \mathrm{~min} ; 35$ cycles of $95^{\circ} \mathrm{C}$ for $30 \mathrm{~s}, 60^{\circ} \mathrm{C}$ for $30 \mathrm{~s}, 72{ }^{\circ} \mathrm{C}$ for $3 \mathrm{~min} ; 72^{\circ} \mathrm{C}$ for $5 \mathrm{~min}$. The detailed information for all the primers is given in Table 2.

\section{Immunofluorescence}

Immunofluorescence experiments were performed as described previously (Zhou et al., 2006). After fixation in $4 \%$ paraformaldehyde in PBS for $30 \mathrm{~min}$ and permeabilization in $0.2 \%$ Triton X-100/PBS for $45 \mathrm{~min}$ at room temperature, all embryo samples were blocked in PBS buffer containing 3\% BSA for $45 \mathrm{~min}$ before incubation with the primary antibodies: anti-GFP antibody (1:1200, Abcam,ab13970) and anti-OCT4 antibody (1:100, Abcam,ab27985) overnight at $4{ }^{\circ} \mathrm{C}$. After rinsing with PBS, embryos were incubated with the secondary antibodies and DAPI in PBS for 
$2 \mathrm{~h}$ at room temperature. EmGFP was labeled with Goat anti-Chicken IgY $(\mathrm{H}+\mathrm{L})$ Secondary Antibody, Alexa Fluor 488 (1:500, Invitrogen, A-11039). Endogenous OCT4 was stained by Donkey anti-Goat IgG $(\mathrm{H}+\mathrm{L})$ Cross-Adsorbed Secondary Antibody, Alexa Fluor 594 (1:500, Invitrogen, A-11058). DNA was counterstained with a DAPI solution (1:500, Solarbio, C0060). Finally, embryos were mounted on glass slides and examined using confocal microscopy.

\section{Off-target analysis}

All potential off-target sites with a degree of homology to the pair of TALENs were predicted by Paired Target Finder in TAL Effector Nucleotide Targeter 2.0 [39] using NCBI GenBank assembly Mmul_051212 (GCA_000002255.2). We chose the 7 highest-ranked off-target sites for analysis. The fragments around these gene sites were amplified using the corresponding primers (Additional file 6) and analyzed by sequencing (Additional file 3 ).

\section{Additional files}

Additional file 1: Supplementary information 1. Sequencing and Blast results of large PCR products of samples 0806.16C1 and 0806.16C2 The sequencing result indicated that EMGFP was successfully inserted into exon 1 of OCT4 as expected. (PDF $1720 \mathrm{~kb}$ )

Additional file 2: Table S1. List of putative off-target sites. The top 7 potential off-target sites (OTS1-OTS7) were selected for analysis. (XLSX 15 $\mathrm{kb})$

Additional file 3: Supplementary information 2. Results of off-target sequencing analysis. (PDF $123 \mathrm{~kb}$ )

Additional file 4: Figure S1. Plasmid profile of the donor vector DonorPKID-E1-EmGFP. (PDF 200 kb)

Additional file 5: Supplementary information 3. Construction of the reporter plasmid for the SSA assay. (PDF $226 \mathrm{~kb}$ )

Additional file 6: Table S2. Primers used for off-target analysis. (XLSX $13 \mathrm{~kb}$ )

\section{Abbreviations}

ASD: Autism spectrum disorder; CRISPR/Cas9: Clustered regularly interspaced short palindromic repeats; DMD: Duchenne muscular dystrophy; DSBs: Double-strand breaks; HD: Huntington's disease; HDR: Homologydirected repair; HR: Homologous recombination; ICM: Inner cell mass; NHP: Non-human primate; PD: Parkinson's diseases; TALENs: Transcription activator-like effector nucleases

\section{Acknowledgements}

We appreciate the technical support for this study by the Shenzhen Cell Inspire Biotechnology. We also appreciate the professional support about animal management and treatment from senior veterinary surgeon Hong Wang.

\section{Funding}

This works is supported by The National Key R\&D Program (2016YFA0101401 and 2016YFC0105703). The former were used for the purchase, raising, and welfare of animals and the funding from the later contributed to experiment in vitro. Besides, the funding bodies were not involved in the design of the study and collection, analysis, and interpretation of data or in writing the manuscript.

\section{Availability of data and materials}

The datasets used and/or analyzed during this study are available from the corresponding author upon reasonable request.

\section{Author contributions}

YYN and WZJ designed the project. CC, ZHY, JYY, LY, CYS, YK, ZZC and YCC performed or analyzed experiments. CC, YYN wrote the paper. All authors read and approved the final manuscript.

\section{Ethics approval}

The cynomolgus monkey (Macaca fascicularis) involved in this study is accredited by AAALAC International and all experimental protocols were approved in advance by the Institutional Animal Care and Use Committee of Yunnan Key Laboratory of Primate Biomedical Research.

\section{Consent for publication}

Not applicable.

\section{Competing interests}

The authors declare that they have no competing interests.

\section{Publisher's Note}

Springer Nature remains neutral with regard to jurisdictional claims in published maps and institutional affiliations.

\section{Author details}

${ }^{1}$ Yunnan Key Laboratory of Primate Biomedicine Research; Institute of Primate Translational Medicine, Kunming University of Science and Technology, Kunming 650500, China. ${ }^{2}$ The Cardiology Division, Department of Medicine, Li Ka Shing Faculty of Medicine, University of Hong Kong, Hong Kong, SAR, China.

Received: 31 July 2018 Accepted: 18 December 2018

Published online: 15 January 2019

\section{References}

1. Izpisua Belmonte JC, et al. Brains, genes, and primates. Neuron. 2015;86(3): 617-31.

2. Niu Y, et al. Generation of gene-modified cynomolgus monkey via Cas9/ RNA-mediated gene targeting in one-cell embryos. Cell. 2014;156(4):836-43.

3. Chen, Y., et al., Modeling Rett syndrome using TALEN-edited MECP2 mutant Cynomolgus monkeys. Cell, 2017. 169(5): p. 945-955.e10.

4. Liu Z, et al. Generation of a monkey with MECP2 mutations by TALEN-based gene targeting. Neurosci Bull. 2014;30(3):381-6.

5. Kang Y, et al. CRISPR/Cas9-mediated Dax1 knockout in the monkey recapitulates human AHC-HH. Hum Mol Genet. 2015;24(25):7255-64.

6. Chan AWS, Chong KY, Martinovich C, Simerly C, Schatten G. Transgenic Monkeys Produced by Retroviral Gene Transfer into Mature Oocytes. Science. 2001:291(12):309-12

7. Yang $\mathrm{SH}$, et al. Towards a transgenic model of Huntington's disease in a non-human primate. Nature. 2008:453(7197):921-4.

8. Chan AW, Yang SH. Generation of transgenic monkeys with human inherited genetic disease. Methods. 2009:49(1):78-84.

9. Nemudryi AA, Valetdinova KR, Medvedev SP, Zakian SM. TALEN and CRISPRCas Genome Editing Systems Tools of Discovery. Acta Nat. 2014;6(3):22.

10. Pu J, et al. Utilization of TALEN and CRISPR/Cas9 technologies for gene targeting and modification. Exp Biol Med (Maywood). 2015;240(8):1065-70.

11. Zhao $\mathrm{H}$, Jiang $\mathrm{YH}$, Zhang $\mathrm{YQ}$. Modeling autism in non-human primates: opportunities and challenges. Autism Res. 2018;11(5):686-94.

12. Ding F, et al. Development of a stable, early stage unilateral model of Parkinson's disease in middle-aged rhesus monkeys. Exp Neurol. 2008; 212(2):431-9.

13. Chen $Y$, et al. Functional disruption of the dystrophin gene in rhesus monkey using CRISPR/Cas9. Hum Mol Genet. 2015:24(13):3764-74.

14. Ousterout DG, Gersbach CA. The development of TALE nucleases for biotechnology. Methods Mol Biol. 2016;1338:27-42.

15. Sakuma T, et al. MMEJ-assisted gene knock-in using TALENs and CRISPRCas9 with the PITCh systems. Nat Protoc. 2016;11(1):118-33.

16. Osborn MJ, et al. TALEN-based gene correction for epidermolysis bullosa. Mol Ther. 2013;21(6):1151-9.

17. Sun $N$, et al. Optimized TAL effector nucleases (TALENs) for use in treatment of sickle cell disease. Mol BioSyst. 2012;8(4):1255-63.

18. Voit RA, et al. Nuclease-mediated gene editing by homologous recombination of the human globin locus. Nucleic Acids Res. 2014;42(2):1365-78. 
19. Ma N, et al. Transcription activator-like effector nuclease (TALEN)-mediated gene correction in integration-free beta-thalassemia induced pluripotent stem cells. J Biol Chem. 2013;288(48):34671-9.

20. Choi SM, et al. Efficient drug screening and gene correction for treating liver disease using patient-specific stem cells. Hepatology. 2013;57(6):2458-68.

21. Shin J, Chen J, Solnica-Krezel L. Efficient homologous recombinationmediated genome engineering in zebrafish using TALE nucleases. Development. 2014;141(19):3807-18.

22. Chu VT, et al. Efficient generation of Rosa26 knock-in mice using CRISPR/ Cas9 in C57BL/6 zygotes. BMC Biotechnol. 2016;16:4.

23. Yang D, et al. Identification and characterization of rabbit ROSA26 for gene knock-in and stable reporter gene expression. Sci Rep. 2016;6:25161.

24. Cui C, et al. Gene targeting by TALEN-induced homologous recombination in goats directs production of beta-lactoglobulin-free, high-human lactoferrin milk. Sci Rep. 2015:5:10482

25. Wu M, et al. Rosa26-targeted sheep gene knock-in via CRISPR-Cas9 system. Sci Rep. 2016;6:24360.

26. Haibo Wu, Y Wang, Yan Zhang, Mingqi Yang, Jiaxing Lv, Jun Liu, and Yong Zhang, TALE nickase-mediated SP110 knockin endows cattle with increased resistance to tuberculosis. PNAS march 2, 2015 p. March 2, 2015.

27. Lai $\mathrm{S}$, et al. Generation of Knock-in pigs carrying Oct4-tdTomato reporter through CRISPR/Cas9-mediated genome engineering. PLoS One. 2016;11(1): e0146562.

28. Yao X, et al. Generation of knock-in cynomolgus monkey via CRISPR/Cas9 editing. Cell Res. 2018;28(3):379-82.

29. Cui Y, et al. Generation of a precise Oct4-hrGFP knockin cynomolgus monkey model via CRISPR/Cas9-assisted homologous recombination. Cell Res. 2018;28(3):383-6.

30. Hockemeyer D, et al. Efficient targeting of expressed and silent genes in human ESCs and iPSCs using zinc-finger nucleases. Nat Biotechnol. 2009; 27(9):851-7.

31. Quan $L$, et al. Establishment of a rabbit Oct4 promoter-based EGFP reporter system. PLoS One. 2014;9(10):e109728.

32. Kosicki M, Tomberg K, Bradley A. Repair of double-strand breaks induced by CRISPR-Cas9 leads to large deletions and complex rearrangements. Nat Biotechnol. 2018; 36(8):765-71.

33. Haapaniemi E, Botla S, Persson J, Schmierer B, Taipale J. CRISPR-Cas 9 genome editing induces a p53-mediated DNA damage response. Nat Med. 2018;24(7):927-30.

34. Yang $\mathrm{H}$, et al. One-step generation of mice carrying reporter and conditional alleles by CRISPR/Cas-mediated genome engineering. Cell. 2013; 154(6):1370-9.

35. Certo MT, et al. Tracking genome engineering outcome at individual DNA breakpoints. Nat Methods. 2011;8(8):671-6.

36. Gu B, Posfai E, Rossant J. Efficient generation of targeted large insertions by microinjection into two-cell-stage mouse embryos. Nat Biotechnol. 2018; 36(7):632-7.

37. Longo PA, et al. Transient mammalian cell transfection with polyethylenimine (PEI). Methods Enzymol. 2013;529:227-40.

38. Niu Y, et al. Transgenic rhesus monkeys produced by gene transfer into early-cleavage-stage embryos using a simian immunodeficiency virus-based vector. Proc Natl Acad Sci U S A. 2010;107(41):17663-7.

39. Cermak $T$, et al. Efficient design and assembly of custom TALEN and other TAL effector-based constructs for DNA targeting. Nucleic Acids Res. 2011; 39(12):e82.

Ready to submit your research? Choose BMC and benefit from:

- fast, convenient online submission

- thorough peer review by experienced researchers in your field

- rapid publication on acceptance

- support for research data, including large and complex data types

- gold Open Access which fosters wider collaboration and increased citations

- maximum visibility for your research: over $100 \mathrm{M}$ website views per year

At BMC, research is always in progress.

Learn more biomedcentral.com/submissions 